\title{
Arquitectura contemporánea en centros históricos: una mirada desde la ciudad de Tunja
}

\author{
Contemporary architecture in historic centers: A view from Tunja
}

Recibido: mayo 2020

Aceptado: febrero 2021

\section{Resumen}

Realizar estudios sobre centros históricos implica hacerse cargo de retos teóricos, históricos y prácticos que dependen de la ciudad objeto de estudio. Acotar correctamente el concepto de centro histórico, entender las dinámicas que lo han transformado con el tiempo y la dificultad para acceder a la información existente, hacen de este tipo de estudios una entrada a nuevas metodologías de análisis y recolección de información para entender las ciudades contemporáneas y sus centros históricos. Tunja tiene un centro histórico valioso, posee importantes edificaciones de los siglos XVI y XVII en muy buen estado, testigos de las transformaciones que ha tenido la arquitectura y el urbanismo durante los últimos quinientos años. Sin embargo, la información que se encuentra es fragmentada y desactualizada. Este artículo presenta la metodología usada para recolectar y organizar datos del centro histórico de Tunja, haciendo énfasis en información urbana $\mathrm{y}$ arquitectónica para inventariar lo existente al tiempo que se rastrean las transformaciones sufridas a lo largo del tiempo. También se busca determinar la forma en que la normativa actual influye en estas transformaciones, y cómo la arquitectura contemporánea se adapta para insertarse dentro del tejido histórico, afectando su contexto, pero sin detener las dinámicas propias del centro.

\section{Palabras Claves:}

centro histórico; metodología; arquitectura contemporánea

\author{
Sebastián Alonso Camargo Castillo ${ }^{1}$ \\ Felipe Andrés Muñoz Cárdenas ${ }^{2}$
}

\begin{abstract}
Conducting studies on historical centers implies taking on theoretical, historical and practical challenges that vary according to the city being studied. From correctly delimiting the concept of a historical center, to understanding the dynamics that have transformed it over the centuries, to the difficulties inherent in doing research, such as the difficulty in accessing existing information, or finding any type of information, these types of studies are gateways to new methodologies of analysis or collection of important information for understanding contemporary cities and the relationship with historical centers. Tunja is no different, it has a valuable historical center insofar as it still has important buildings from the 16th and 17th centuries in a good condition and it is possible to trace in its historical center traces of the transformations that architecture and urban planning have undergone over the last five hundred years. However, the information found is fragmented and, in many cases, outdated. This article presents the methodology used to collect and organize data from the historical center of the city, emphasizing urban and architectural information that allows for an inventory of what exists while tracking the transformations suffered over time. Similarly, it seeks to determine the way in which current regulations have determined these transformations, and how contemporary architecture has adapted to insert itself into the historical chain, affecting its context, but without stopping the urban dynamics of the city center.
\end{abstract}

\section{Keywords:}

historic center; methodology; contemporary architecture

\footnotetext{
1 Nacionalidad: colombiano; adscripción: Universidad Santo Tomás Tunja; Magister en Historia y Teoría del Arte, la Arquitectura y la ciudad; correo: sebastiancamargoc16@gmail.com

2 Nacionalidad: colombiano; adscripción: Universidad Santo Tomás Tunja; Dirección de Investigación e Innovación, correo: felipe.munoz@ usantoto.edu.co
} 


\section{INTRODUCCIÓN}

La ciudad de Tunja tiene uno de los centros históricos más antiguos de Colombia. Si bien su fundación hispánica se da el 6 de agosto de 1539, la ciudad también tiene una rica historia prehispánica de la cual hay muy pocos vestigios porque no se conocen con certeza los detalles del poblamiento de esta parte del continente y porque los hallazgos arqueológicos rara vez contienen evidencias arquitectónicas (Arango, 2019). Debido a lo que aún se conserva, la ciudad es considerada una joya de la colonia gracias a inmuebles representativos de la arquitectura doméstica y religiosa del siglo XVI y XVII entre los que se encuentran, la Casa de Fundador, la casa Holguín, la casa Ruíz Mancipe, y diferentes iglesias pertenecientes a las primeras parroquias de la ciudad como Santa Bárbara, Santo Domingo y la Catedral. Además de las edificaciones, en Tunja la traza ortogonal conformada desde su fundación se conserva, adaptada a la fuerte topografía de su emplazamiento y, aún hoy, es el elemento estructurador del centro histórico.

El perímetro de la ciudad durante los siglos XVI, XVII y XVIII fue declarado como sector antiguo con la ley 163 de 1959, considerada la primera ley de protección del patrimonio en Colombia. Con el paso de los años y las nuevas necesidades urbanas, la legislación colombiana ha evolucionado con miras a desarrollar instrumentos de regulación de las intervenciones que se hacen en las edificaciones monumentales y su contexto.

En el año 2008, se crea la figura de Planes Especiales de Manejo y Protección(PEMP) que "es el instrumento de gestión del patrimonio cultural por medio del cual se establecen las condiciones necesarias para garantizar su proyección y sostenibilidad en el tiempo" (Ley 1185, 2008). Debido a los antecedentes y la importancia que tiene el centro de Tunja junto con las urgentes necesidades de mejoramiento y salvaguardia de su patrimonio construido, en 2012, se aprueba el Plan Especial de Manejo y Protección del Centro Histórico de Tunja y su Zona de influencia.

Este PEMP, en su momento diagnosticó el estado del centro y propuso estrategias normativas, proyectuales y de gestión que debían ser implementadas para acometer su objetivo general, "Recuperar y exaltar integralmente el patrimonio cultural del Centro Histórico de Tunja y recalificar su territorio en los diversos aspectos urbanos para garantizar su continuidad como principal centralidad de la ciudad" (Resolución 0428, 2012). Así mismo, establece también las condiciones que debe cumplir una obra nueva que se construya dentro de la delimitación del área afectada y su zona de influencia.

Este trabajo nació con el objetivo de desarrollar una metodología para la recolección de datos, tanto históricos como actualizados, que permitieran tener un panorama más aproximado de las dinámicas urbanas que afectan el patrimonio arquitectónico de la ciudad y que sirviera como el primer insumo para la creación del Observatorio Urbano del Centro Histórico de Tunja. Sin embargo, en este artículo se presenta la metodología aplicada para la recolección y organización de la información $\mathrm{y}$ algunas reflexiones que han surgido como resultado de entender que el centro histórico no es un sitio detenido en el tiempo sino por el contrario, es el espacio donde están todos los momentos, incluida nuestra contemporaneidad, y la forma en que esta se ha adaptado a la norma arquitectónica vigente $\mathrm{y}$ ha transformado parte importante de ese patrimonio, adaptándolo, aumentándolo, copiándolo o sencillamente destruyéndolo.

Las construcciones contemporáneas y la forma en que estas se incrustan en los centros históricos plantean una discusión de gran importancia si se consideran las implicaciones en el contexto urbano que éstas tienen. El centro de Tunja como muchos otros en el Colombia, no se ha desarrollado de una manera planificada, sino que ha sido resultado de la adición de momentos históricos representados en la construcción de edificaciones que son la respuesta que cada generación ha venido dado a su momento histórico en particular. Esta condición constituye un testimonio edificado que permite transmitir a través de su observación y análisis la huella de cada uno de los momentos históricos que han transcurrido en lo que hasta mediados del siglo XX fue la totalidad de la ciudad.

\section{LA CONTEMPORANEIDAD DEL CENTRO DE TUNJA}

Las herramientas tecnológicas vigentes ofrecen nuevas alternativas para una gestión urbana más eficiente de los centros históricos. Por desgracia, en Tunja no existe ningún organismo, entidad o institución que se dedique a la recolección, registro y evaluación de los fenómenos urbanos de la ciudad. 
Si bien dentro de la correspondiente aprobación de cualquier intervención o construcción en el centro histórico existen organismos que velan por la aplicación de la normativa del PEMP, existen casos en los cuales es importante tener referencias históricas y datos urbanos más precisos para la toma de decisiones, elementos que no existen y que propician que sea el criterio del funcionario de turno el que determine la decisión sobre un proyecto arquitectónico. Si bien en el momento de la formulación y aprobación de los proyectos la información necesaria es escasa, durante su ejecución el proceso de vigilancia es prácticamente nula, los propietarios de los proyectos realizan obras en horas nocturnas, en días festivos, o comienzan las obras de puertas para adentro sin los permisos necesarios para evadir la actuación de las autoridades, quienes dependen casi que exclusivamente de las denuncias ciudadanas.

Registrar y organizar digitalmente los fenómenos de la realidad del centro, que incluya sus dinámicas urbanas, las transformaciones y afectaciones de los monumentos y todo lo que de esto se desprende permite contar con un insumo valioso tanto para la evaluación constante que fundamenta las decisiones de las administraciones como para una amplia divulgación que permita mayor reconocimiento de la comunidad con su patrimonio.

En medio del vacío de información y la pérdida de la valiosa oportunidad de registrar los fenómenos contemporáneos en tiempo cercano a su ocasión, un paso fundamental es aportar al análisis de las nuevas edificaciones para evaluar sus repercusiones en el contexto urbano.

En la actualidad, el centro de Tunja alberga las principales funciones de entidades administrativas, educativas, comerciales, turísticas y culturales de la ciudad. Así mismo, congrega las principales manifestaciones sociales, culturales y religiosas, incluyendo la celebración de la Semana Santa, patrimonio cultural inmaterial de la nación (Ley 1767, 2015), que forma parte importante de las tradiciones vigentes.

Lo que hoy consideramos centro histórico, fue también "la ciudad toda" (Carrión, 2010), es decir que, desde su fundación hasta casi mediados del siglo XX, atendió todas las necesidades de su momento, que luego con el tiempo, se han transformado para atender a las generaciones presentes, y por ser el centro, recibe en su transcurrir el vaivén y el impacto de todos los cambios sociales y espaciales (Aprile-Gniset, 2010)
En el siglo XX, la ciudad crece, surgen nuevos polos de desarrollo y el centro tiene la necesidad de reformarse en función de la nueva demanda de servicios y con una idea del denominado progreso implantada en la población. Eso incluyó alteraciones de todo tipo en los inmuebles, e inclusive demoliciones para la construcción de nuevos y representativos edificios en altura. Influyeron múltiples factores: la llegada del automóvil, el surgimiento de nuevos tipos de comercio y establecimientos, que junto con el carácter religioso y administrativo que ya tenía, construyeron una nueva idea del centro de Tunja.

La construcción de nuevas edificaciones es el resultado de la necesidad manifiesta que tiene el centro de revitalizarse para atender la demanda de vivienda y servicios, en esa medida, las repercusiones que estos conllevan deben ser evaluadas en el corto plazo, con el único fin de contribuir en una mayor conservación del contexto patrimonial. Por lo anterior, es importante acotar una definición de la arquitectura contemporánea de los centros históricos.

"proceso proyectual subjetivo y actual de evolución, sin delimitación de tiempo específico, sin espacialidad definida, dinámica y sin "sello" histórico denominado; desde la relatividad, es una producción material e inmaterial que conjuga las actuales dinámicas espaciales y culturales para ser parte del entorno, que aspira relacionar armónicamente contextos, formas, funciones $\mathrm{y}$ materialidad de manera sistémica para beneficio colectivo; un presente coyuntural entre lo preexistente y el porvenir." (Pasuy, 2015:6)

Las nuevas construcciones intervienen en la definición del paisaje urbano, para Choay (2007) "en lo que respecta a la modernización del tejido urbano antiguo, ésta se realiza llenando los vacíos existentes o creados para tal efecto." (p. 205). La autora recalca también una condición fundamental que debería tener la arquitectura contemporánea, "Los elementos arquitectónicos modernos (o posmodernos) reputados como aportaciones valorizantes de la ciudad antigua, lo son, efectivamente, a condición de haber respetado tanto esa articulación como sus reglas morfológicas pero no lo son, como a menudo es el caso, cuando operan en tanto que objetos independientes y autosuficientes." (Choay, 2007:205)

Sumado a lo anterior, el Plan Especial de Manejo y Protección de 2012, es una variable adicional para el análisis porque aunque la 
arquitectura contemporánea no puede ser definida en tiempo o espacio por su condición natural de estar construyendo el presente, es el instrumento normativo el que regula a partir de su creación, las condiciones urbanas y arquitectónicas mínimas que deben tener las nuevas edificaciones en el centro de Tunja en aspectos tales como:

- Volumetría: Altura entrepisos, número de pisos, empates, aislamientos, voladizos, paramentos, patios.

- Materialidad: Cubiertas, fachadas.

- Uso: Comercial, residencial, servicios e institucional.

Dentro de los anteriores aspectos surge una importante premisa que se plantea en el artículo 72 del PEMP, "Se pueden hacer intervenciones contemporáneas de los valores tradicionales de la arquitectura tunjana, utilizando lenguajes actuales que no generen falsos históricos o lenguajes antiguos." (Resolución 0428, 2012) que de cierta forma deja abierta la posibilidad a un sinfin de interpretaciones, que parten de la definición de los valores tradicionales, la selección de un lenguaje y su respectiva aplicación en las nuevas construcciones permitidas.

De esta manera, el análisis de las nuevas construcciones del centro histórico debe considerar no sólo los aspectos teóricos de diseño sino también, los normativos y, además, su influencia en la caracterización de la arquitectura y el impacto producido en el contexto.

\section{METODOLOGÍA PARA LA RECOLECCIÓN DE INFORMACIÓN}

La recolección de la información urbana en los centros históricos, se ha desarrollado desde que se consideró la necesidad de identificar, documentar y conservar el patrimonio inmueble en Colombia. En ese sentido, los ejercicios pioneros propuestos por Alberto Corradine, Germán Téllez, Ernesto Moure, entre otros ${ }^{3}$ impulsaron la creación de herramientas de reconocimiento de las edificaciones más representativas de cada ciudad. Así pues, en la actualidad, el Ministerio de Cultura se ha encargado de evolucionar en estrategias como bien está representado en el "Manual para Inventario de Bienes Inmuebles" (Ministerio de Cultura, 2005)

En ese contexto, las condiciones ya planteadas por todos los trabajos realizados, sientan un precedente en el análisis individual de los inmuebles y en algunos casos de perfiles de manzana. Sin embargo, no son útiles para el reconocimiento ágil de características meramente urbanas y para convertirse en un insumo que pueda ser administrado a través de los sistemas de información geográfica utilizados en la actualidad. En esa medida, se consideraron los siguientes elementos para conformar una ficha que, a partir del recorrido, fuente primordial de recolección de datos, permitiera sistematizar la información y, así mismo, garantizar que esta pueda ser actualizada frecuentemente una vez sea vinculada al sistema de forma ágil.

Figura 1. Estructura ficha de recolección de información

Fuente: Elaborada por los autores

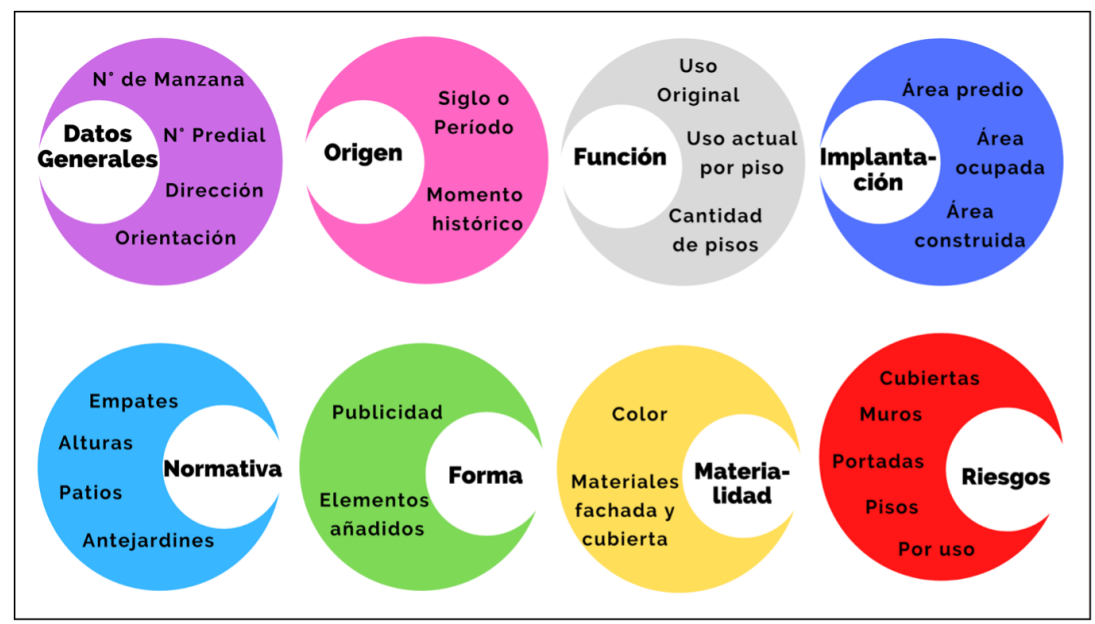

\footnotetext{
${ }^{3}$ Los estudios analíticos y reglamentarios encargados por la Corporación Nacional de Turismo en 1980 así como los estudios de 1973 del arquitecto Corradine incluidos en el Plan de Conservación Nacional hacen parte de la gran variedad de propuestas y proyectos ejecutados desde mediados del siglo XX.
} 
Esta ficha permite la recolección de información de un momento específico y permite hacerse una idea de la situación arquitectónica del centro de la ciudad, sin embargo, para que sea útil, se debe establecer no solo una periodicidad en la recolección de la información, que para este caso es cada tres meses, sino que se deben plantear estrategias para la recolección de información histórica que nutra el proceso evolutivo de cada predio del área de estudio de tal forma que la información de la que dispongan los investigadores, los proyectistas y las autoridades sea no solo actualizada, sino histórica para facilitar la toma de decisiones fundamentadas. Toda la información recolectada es ingresada en un sistema de información geográfico que permite organizar, y acceder a la información de forma más efectiva y con la posibilidad de hacer matrices con distintas fuentes de información. En principio y debido a la disponibilidad del grupo de trabajo, se tuvieron en cuenta los criterios arquitectónicos referidos en la figura 1, sin embargo es necesario ampliar los criterios que se tienen en cuenta en la recolección de información, de tal forma que a los ya relacionados se sume información social, ambiental, histórica, cultural, etc... que permitan alcanzar niveles de análisis urbanos más completos para la toma de decisiones no solo normativas urbano - arquitectónicas sino de políticas públicas de ocupación del espacio y prospectiva del centro histórico de la ciudad.

Los sistemas de información geográfica cumplen un rol sustancial en la localización de los predios con características específicas que, para este caso, son aquellos que fueron sujeto a proceso de las licencias de construcción después de la aplicación del PEMP en el 2012. La herramienta permite reducir el tiempo empleado en recorridos, y evaluar en escalas temporales, determinadas previamente, los inmuebles sujetos de análisis. Además, gracias a este software, la información recolectada puede ser organizada individualmente por predio para evaluar sus repercusiones urbanas, crear un acervo fotográfico histórico y ser insumo para análisis de otros aspectos, como el económico o el ambiental.

La creación de las bases de datos para administrar la información a través de los sistemas de información geográfica se hace de manera conjunta entre el recorrido y un análisis posterior utilizando el registro fotográfico. Así pues, la condensación de los datos enlistada y organizada se convierte en material que permite diagnosticar y evaluar de forma sintética los fenómenos urbanos del centro y a su vez, ser de una fácil divulgación entre la población.

Figura 2. Ficha de levantamiento de información urbana (parte 1)

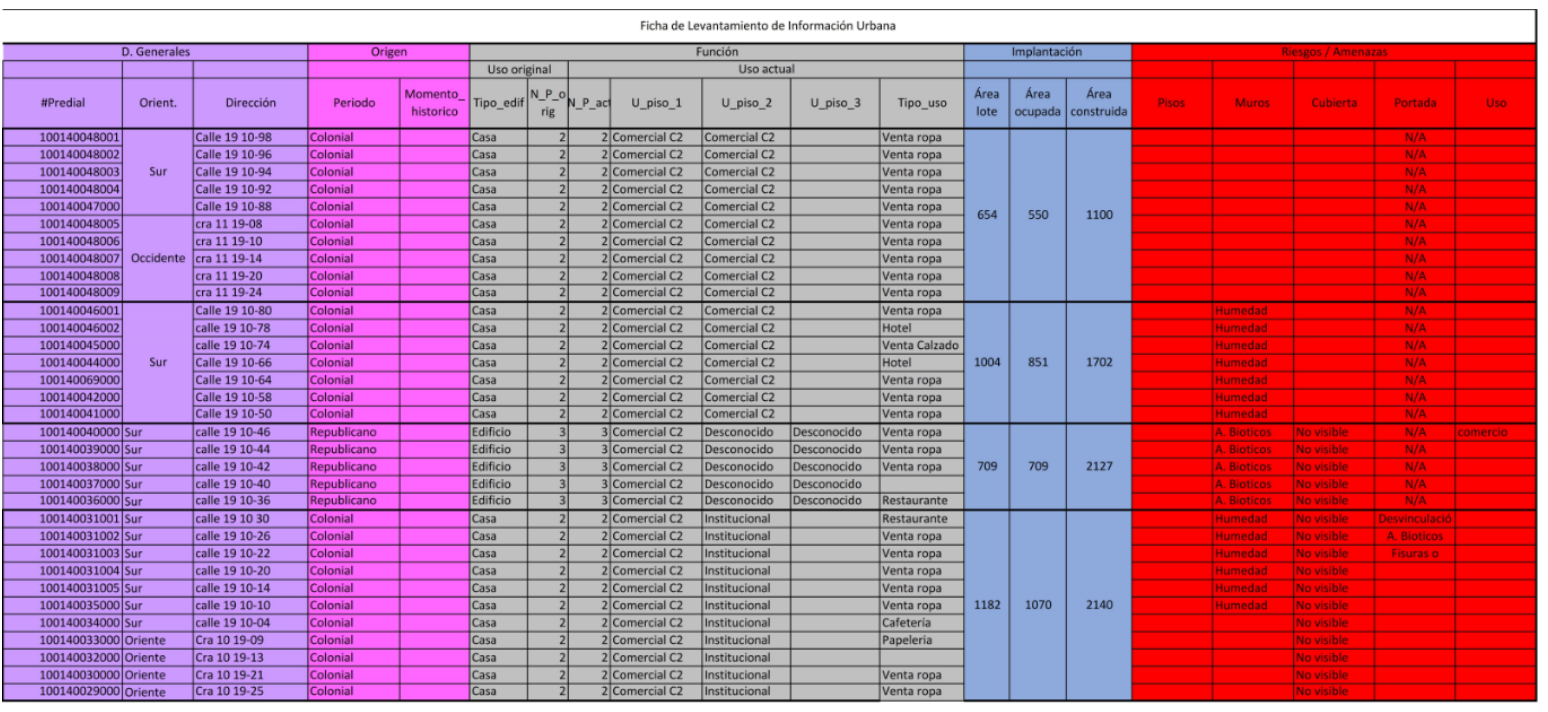

Fuente: Elaborada por los autores 
Figura 2. Ficha de levantamiento de información urbana (parte 2)

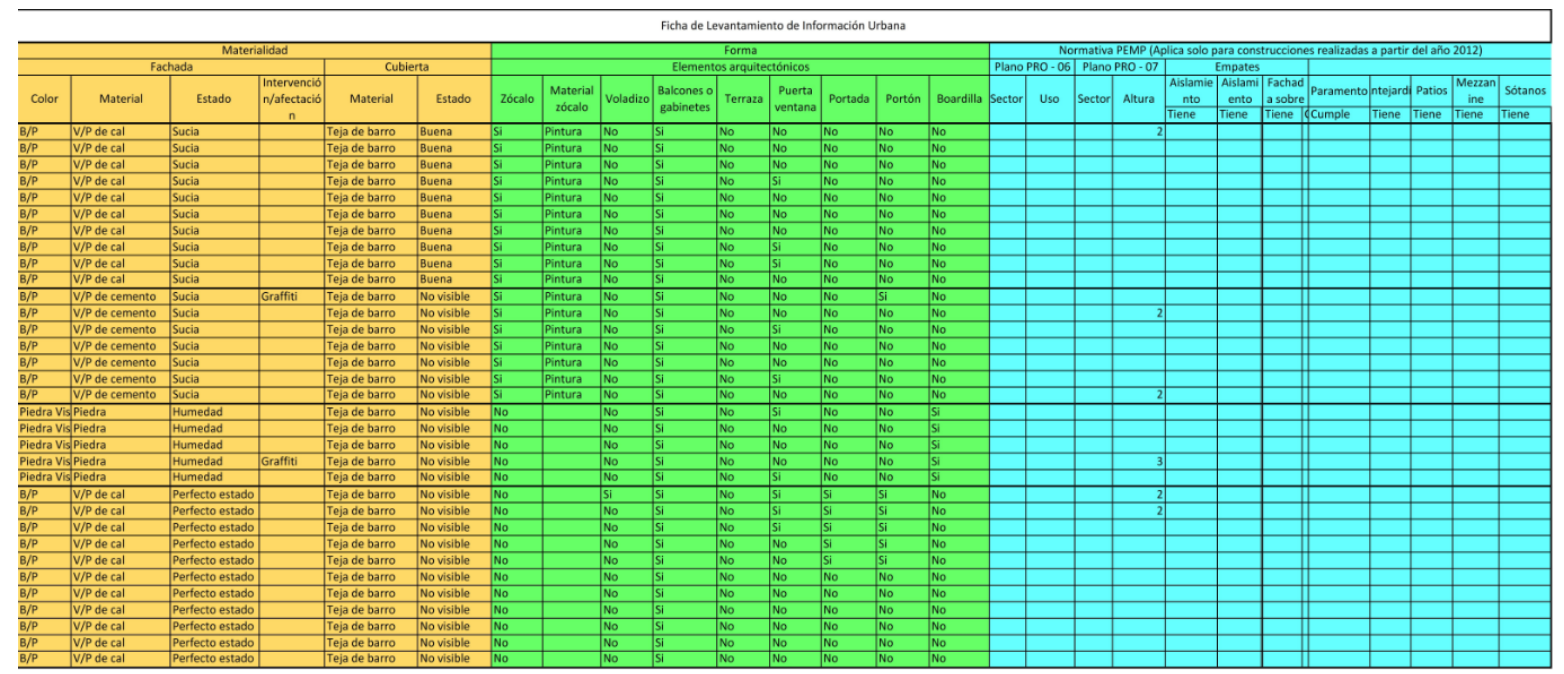

Fuente: Elaborada por los autores

La siguiente imagen es un ejemplo de un mapa exportado gracias a la recepción y/o creación de bases de datos en un Sistema de Información Geográfica. Allí se presenta el perímetro establecido por el PEMP para el centro histórico y su área de influencia, se relacionan los inmuebles de categoría nivel 1 , es decir, aquellos cuyas características denotan gran importancia de carácter histórico, estético o simbólico. También, se registran los inmuebles nivel 2 que son conformados por los inmuebles cuya valoración tiene elementos de menor de importancia y, por último, en color amarillo, todas las construcciones realizadas desde el año 2012.

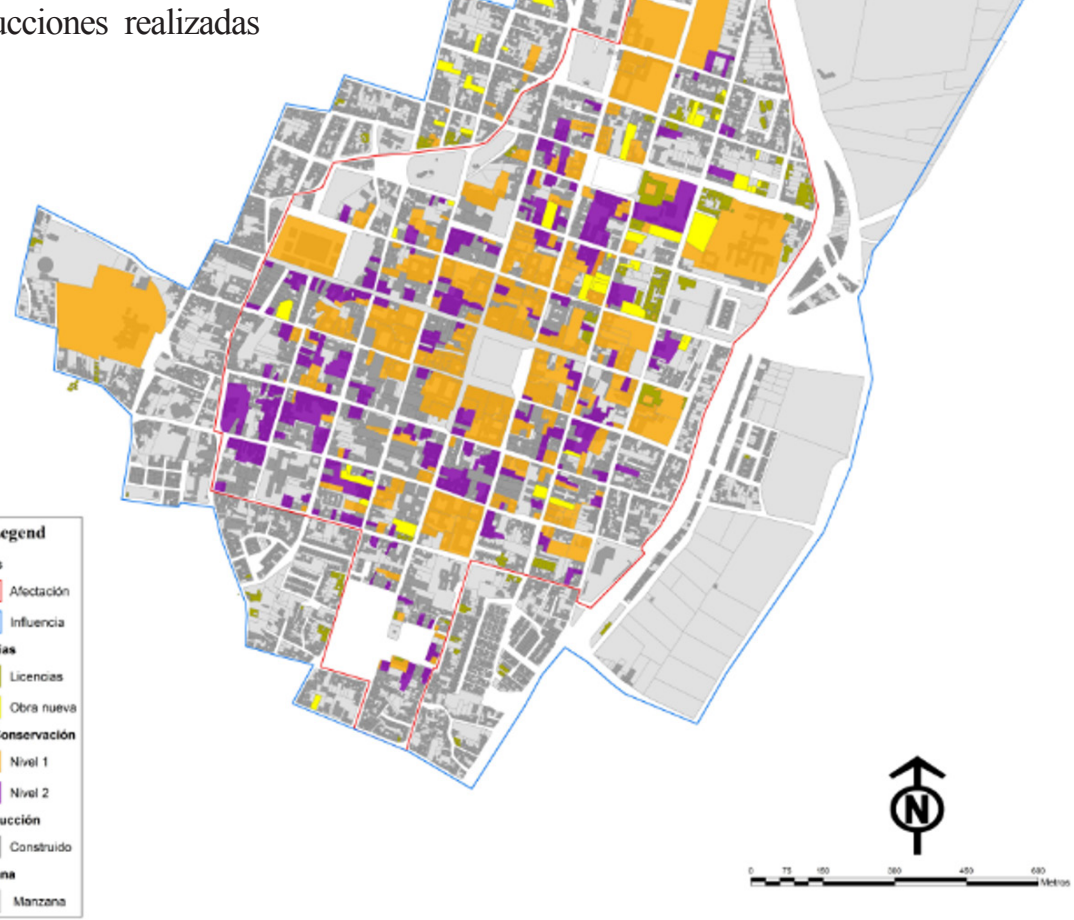


Con este proceso se logra recolectar altos volúmenes de información, administrarlos a través de una única plataforma para así realizar los análisis correspondientes dependiendo de los intereses que se tengan. En ese sentido, todos los datos ilustrados de manera gráfica en el mapa son fácilmente cuantificables y susceptibles de ser relacionados con nueva información recolectada dando la versatilidad requerida para el análisis de variables de cualquier tipo.

\section{ANÁLISIS}

Una primera aproximación a la lectura de la información se realizó a partir de la identificación de diversas formas de arquitectura contemporánea y su relación con la normativa PEMP vigente. Para abordar el análisis de las edificaciones halladas, debe considerarse la posibilidad que tienen las construcciones contemporáneas de contrastar o adaptarse a su respectivo contexto, sin el ánimo de emitir un juicio sobre la validez de una u otra, sino de poner en valor las connotaciones que estas tienen urbanamente y ejemplificar estas posibilidades a través de lo que está ocurriendo en Tunja.

De esa manera se abre el lente y no se descarta ninguna de las posibles manifestaciones. Un resultado preliminar es la gran similitud entre las construcciones, la posibilidad de establecer características que son comunes indistintamente de su localización en el centro histórico y que conlleva a considerar la existencia de condicionantes en los diseños que marcan una impronta de nuestra contemporaneidad.

Preliminarmente, como una aproximación inicial del análisis propuesto, se encuentran cuatro categorías de edificaciones con características similares que permiten organizarlos de la siguiente manera:

\section{GRUPO 1}

Figura 4. Edificaciones contemporáneas (grupo 1)

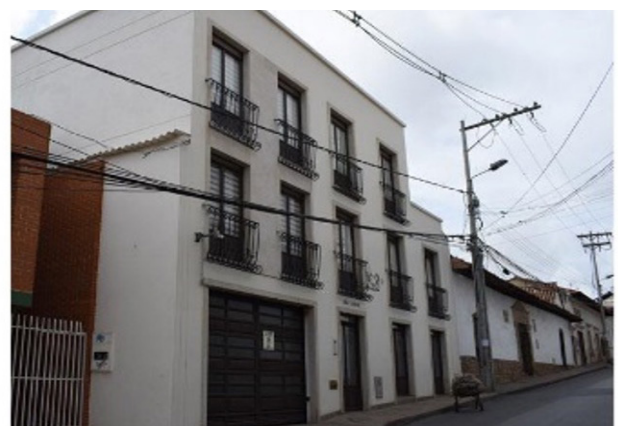

Figura 4. Edificaciones contemporáneas (grupo 1)
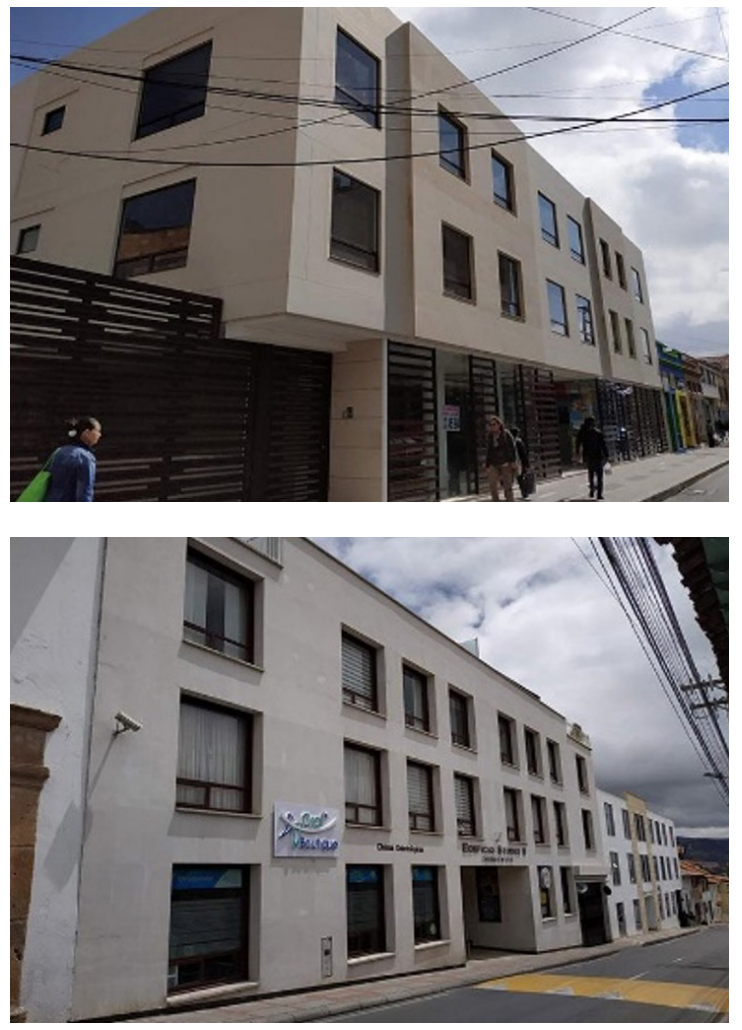

Fuente: Fotografías por los autores

En primer lugar, se encuentran las edificaciones que responden a la demanda de vivienda nueva en el centro de la ciudad. Son mayoritariamente de uso mixto, comercio en primer piso y vivienda en los pisos siguientes y su distribución es similar a las condiciones de vivienda contemporánea en el resto de la ciudad. A nivel formal, recurren a la repetición y regularidad de vanos en fachada. Poseen también materialidades austeras como el enchape con láminas de piedra arenisca y el uso de colores claros.

\section{GRUPO 2}

Figura 5. Edificaciones contemporáneas (grupo 2)

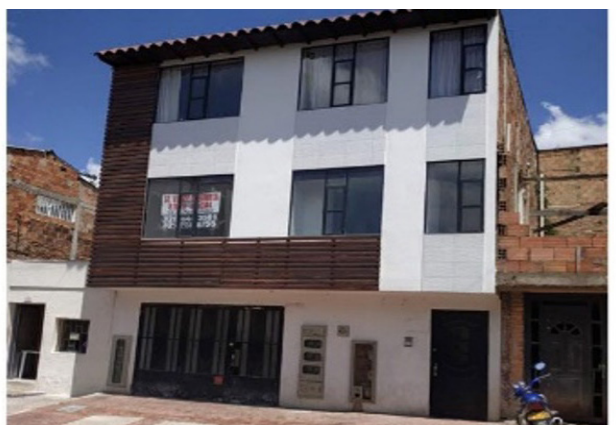


Figura 5. Edificaciones contemporáneas (grupo 2)
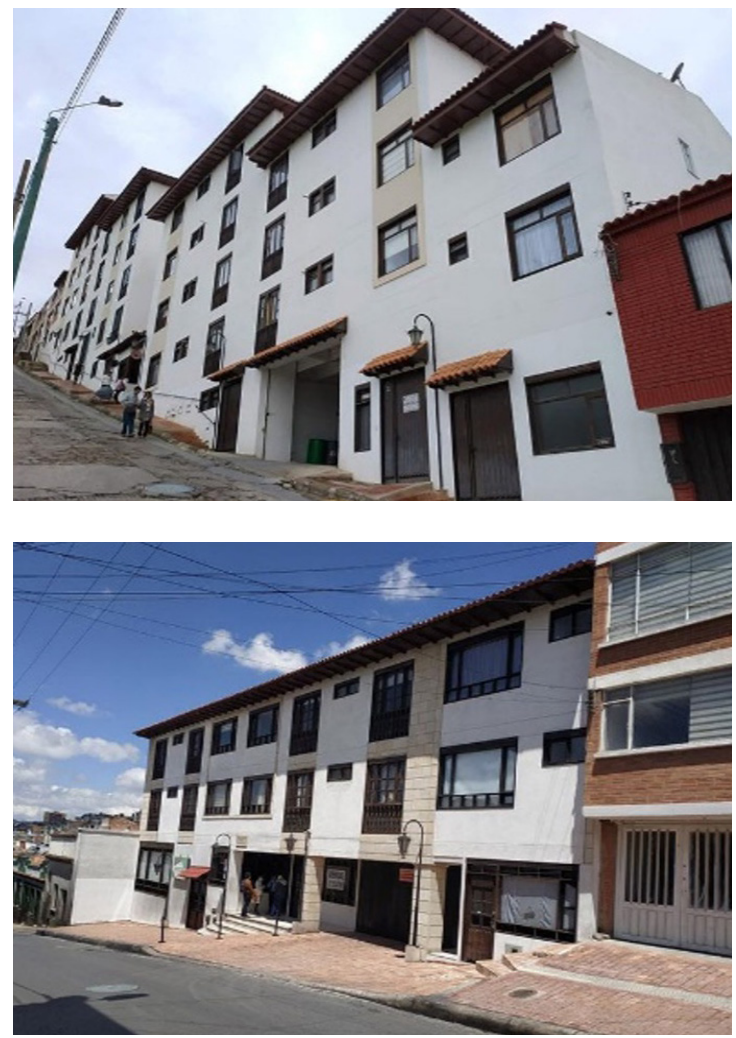

Fuente: Fotografias por los autores

En un segundo grupo están las edificaciones que, aunque también son de carácter residencial, poseen características formales un tanto distintas al primero. Su estrategia se basa en el uso de materiales para aparentar los utilizados en las casas coloniales y republicanas del centro. Se acude al uso de tejas de barro, marcos en madera, portones y barandas en forja, pero poseen alturas que varían entre 3 y 5 pisos.

\section{GRUPO 3}

Figura 6. Edificaciones contemporáneas (grupo 3)

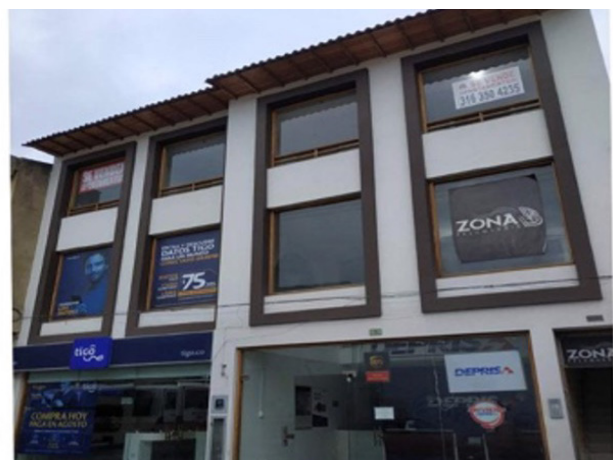

Figura 6. Edificaciones contemporáneas (grupo 3)
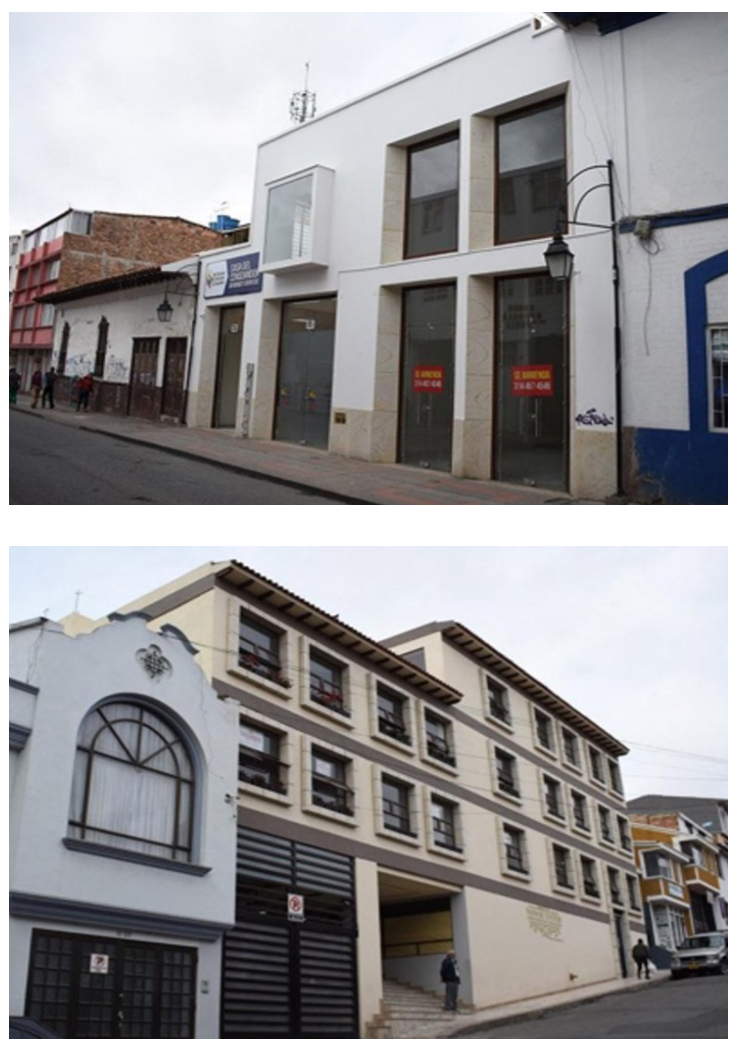

Fuente: Fotografias por los autores

Esta categoría está conformada solamente por las edificaciones que tienen uso comercial puesto que conllevan dinámicas distintas. Se conforman por locales comerciales y oficinas que configuran espacios interiores diferentes a los de uso residencial pero que, a pesar de eso, poseen fachadas de gran similitud a las de carácter residencial.

\section{GRUPO 4}

Figura 7. Edificaciones contemporáneas (grupo 4)

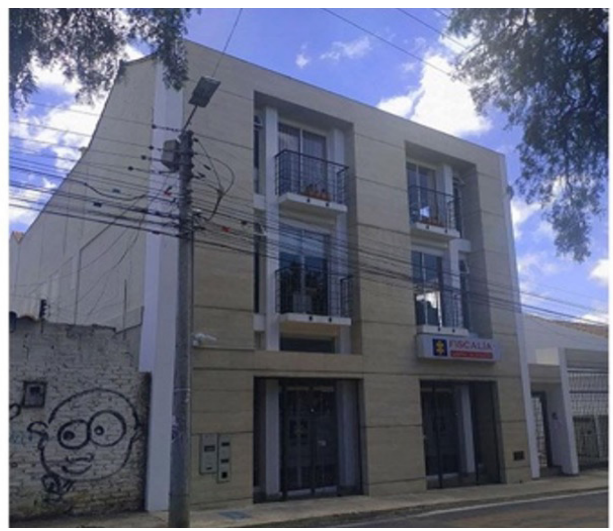


Figura 7. Edificaciones contemporáneas (grupo 4)
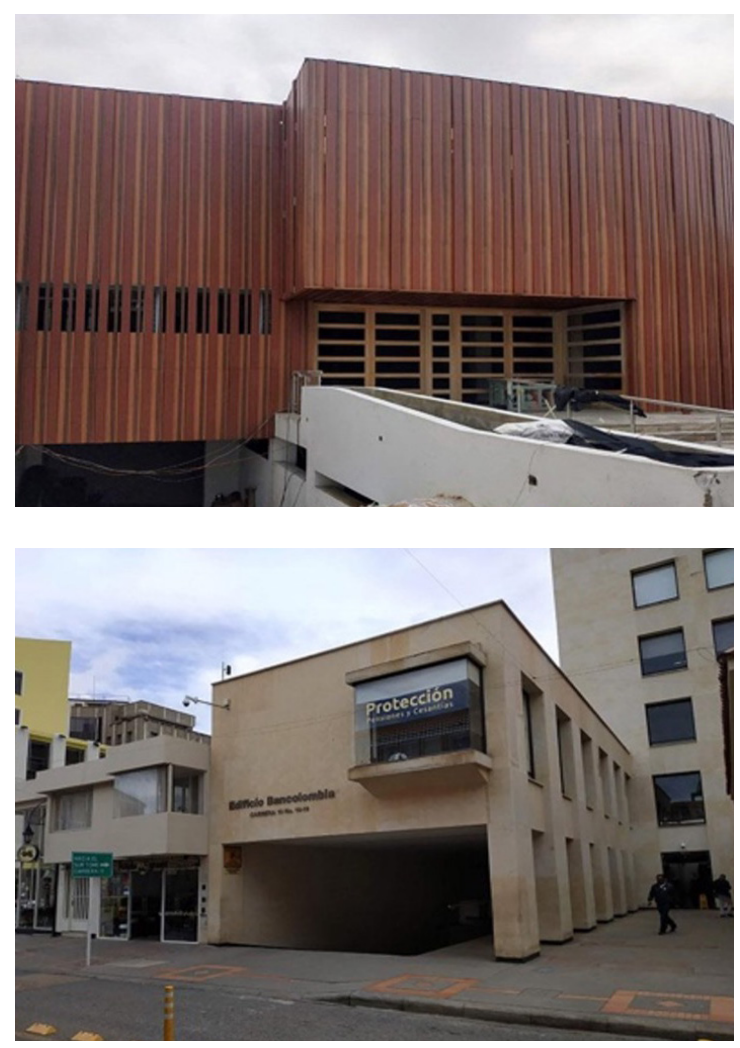

Fuente: Fotografias por los autores

Este grupo concentra aquellas edificaciones que responden a necesidades culturales o institucionales. Se podría asumir que esa variedad de usos implica asimismo una variedad de estilos arquitectónicos pero contrario a eso, las respuestas formales y materiales son similares en la mayoría de casos, a las contenidas en los grupos anteriores.

Sin importar el grupo en que se clasifiquen las edificaciones, éstas poseen algunas características comunes. Estacionamientos privados; ausencia de voladizos; predilección por los colores claros; geometrías rígidas; y una proporción equitativa de vanos hacen ver una inquietante correlación formal y material, casi como un patrón, en cada grupo.

En principio, debe considerarse que esa condición de similitud corresponde por una parte a las presiones ejercidas por el mercado inmobiliario, el valor del suelo en el centro es elevado a razón de la aún imperante condición jerárquica de usos comerciales, educativos e institucionales. En un segundo plano, no menos importante, la regulación del PEMP y los instrumentos diseñados para la aprobación de proyectos nuevos influyen sustancialmente debido a la rigurosidad con la que, tanto las curadurías urbanas, como la Comisión del Centro Histórico, aplica en cualquier proyecto que se plantee.

En este sentido, se debe profundizar en algo que gracias a lo recolectado es evidente, las edificaciones contemporáneas del centro de Tunja son una réplica unas de otras. El escenario es negativo al considerar que estas edificaciones parecieran ser el resultado de la aplicación de una fórmula, producida por el mercado inmobiliario, y adaptada a la norma, con la cual se obtiene siempre el mismo resultado, es decir, la aprobación del permiso para construir.

Ante este escenario, surgen algunas preguntas que harían parte de otros niveles de análisis de la información recolectada, ¿la norma PEMP es tan restrictiva que no permite otro tipo de expresión arquitectónica?, ¿no están en capacidad los arquitectos de encontrar nuevas formas de contextualización histórica aun con la normativa PEMP? o ila premura por comenzar proyectos y la presión inmobiliaria hacen que se vuelva una y otra vez a la fórmula que funciona?, ¿es posible lograr nuevos tipos de apropiación arquitectónica con la norma actual?. Esto teniendo en cuenta que, en todos los casos de intervenciones arquitectónicas en el centro histórico de la ciudad, se hayan seguido estrictamente las determinantes de la norma.

\section{CONCLUSIONES}

A partir de la información recolectada y con el ánimo de incrementar un acercamiento a los fenómenos contemporáneos que suceden en los centros históricos, existen ciertas conclusiones tempranas.

La consideración del uso de Sistemas de Información Geográfica como herramienta de gestión urbana y de divulgación de la información es ciertamente válida en tanto que, a través de éste, los procesos y análisis de la información fueron mucho más ágiles. Además, el trabajo realizado al respecto es base sustancial para futuros adelantos que se quieran dar con miras a ampliar la evaluación de dinámicas actuales, para que mandatarios y técnicos tomen mejores decisiones en pro de la conservación del patrimonio. También, gracias a los datos recogidos y organizados se desprenden nuevos interrogantes, por ejemplo, la condición restrictiva de los límites urbanos establecidos por el PEMP y el análisis de las transformaciones arquitectónicas que suceden en los inmuebles del centro a manera de adiciones, sustracciones o 
transformaciones que repercuten en la preservación de los edificios y las alteraciones que estos sufren.

En segunda instancia, debe considerarse las implicaciones que producen las normativas actuales en los centros históricos. En Colombia los Planes Especiales de Manejo y Protección han sido implementados en 25 ciudades (Ministerio de cultura, 2017), por lo cual no deja de ser motivo de inquietud evaluar si estos instrumentos junto con los órganos encargados de aprobar las intervenciones están ultimando los proyectos de arquitectura en los centros históricos a la constante repetición formal que cercena la posibilidad de plasmar lenguajes propios y de tener acceso al derecho de la variedad de la arquitectura y la sociedad.

La arquitectura contemporánea del centro de Tunja es producto de la necesidad de vivienda y comercio que se ha mantenido vigente gracias al carácter de centralidad que valientemente no ha perdido a lo largo del tiempo. La existencia de nuevos centros urbanos que concentran actividades representativas de la idea de "progreso" implantada en nuestra sociedad; centros comerciales, médicos o financieros, no han producido una pérdida significativa de la importancia funcional del centro histórico.

Por lo anterior, la ruta ideal para mantener la función central que tiene la parte más antigua de la ciudad, debe estar orientada a reforzar la existencia de equipamientos que sirvan a las necesidades de los habitantes permanentes como son las panaderías, zapaterías, minimercados, etc, que permitan tener condiciones adecuadas para un modelo de vida contemporáneo sin perjudicar las tradiciones instauradas, sino al contrario, reforzando la condición de cotidianidad que éste requiere.

En otro sentido, debe considerarse si los ejercicios proyectuales se insertan en su contexto y responden a las condiciones singulares de este, o si, como en el caso de Tunja, estos parecen ser sacados únicamente con el fin de responder económicamente a las condiciones inmobiliarias y se alejan de la obligación ética que tiene la arquitectura en contextos patrimoniales de responder a las condiciones de su entorno. $\mathbf{C}$

\section{REFERENCIAS BIBLIOGRÁFICAS}

APRILE-GNISET, Jacques (2010), La ciudad Colombiana, Cali, Universidad Del Valle.

ARANGO, Silvia (2019), Historia de la arquitectura en Colombia, Bogotá, Universidad Nacional de Colombia.

CARRIÓN, Fernando (2010), El laberinto de las centralidades históricas en América Latina, Quito, Ministerio de Cultura del Ecuador. Recuperado de https://bit.ly/2zbqCJG

CHOAY, Françoise (2007), Alegoría del patrimonio, Barcelona, Editorial Gustavo Gili.

Ley 1185. Congreso de la República, Bogotá, Colombia, 12 de marzo de 2008. Recuperado de https://bit.ly/3c7uIBj

Ley 1767. Diario oficial, Bogotá, Colombia, 07 de septiembre de 2015. Recuperado de https://bit.ly/2zbrqyc

Ministerio de Cultura. (2005), Manual para inventarios Bienes Culturales Inmuebles, Bogotá, Ministerio de Cultura.

Ministerio de Cultura de Colombia. (2017). Políticas, planes y programas. Recuperado de https://bit.ly/2W9sHz0

PASUY, William (2015), "Centro histórico y arquitectura contemporánea: Aproximación teórica para el siglo XXI", Revista M, Vol. 12. diciembre, pp. 52-61. Doi: https://doi. org/10.15332/rev.m.v12i1.1926

Resolución 0428. Ministerio de Cultura de Colombia, Bogotá, Colombia, 27 de marzo de 2012. Recuperado de https://bit. ly/2SEqUiZ 